\title{
THE TRAINER PROJECT: A NEW SIMULATOR-BASED DRIVER TRAINING CURRICULUM
}

\author{
Juan F. Dols \\ J. Pardo \\ Mechanical and Materials Engineering Department \\ UPV, Polytechnic University of Valencia (SPAIN) \\ E-mail: jdols@mcm.upv.es
}

T. Falkmer

VTI, Swedish National Road and Transport Research Institute, Sweden

E. Uneken

RUG, University of Groningen, The Netherlands

W. Verwey

IFADO, Institute for Occupational Physiology IfADo, Germany

\begin{abstract}
Summary: The purpose of the EU funded TRAINER project is to develop a new cost-effective Pan-European driver training curriculum, including computer-based interactive multimedia and simulator technology. The curriculum will pay significant attention to higher order skills including risk awareness. For this purpose a number of scenarios were developed that addresses the most important needs of learner drivers. These scenarios are used in a PC-based interactive multimedia tool as well as in a driving simulator. The interactive multimedia tool allows training and assessment of higher cognitive skills (i.e., strategic and manoeuvring tasks), familiarisation of novice drivers with the basic principles of driving, and contributing to a better understanding of (potential) risks. A low cost stationary driving simulator is used for acquiring skills in vehicle handling and negotiating common traffic situations (i.e., manoeuvring and control tasks). In addition, a mean cost semi-dynamic driving simulator is developed for supporting the needs of specific driver cohorts, such as novice drivers with enhanced knowledge problems and drivers in high-risk groups. Application of such an advanced computer-based curriculum also implies development of criteria to allow driving instructors to determine training progress. These criteria are based on a database of normative driver behaviour. This paper mainly focuses on the description of the technical (softand hardware) requirements for both low-cost and mean-cost simulators.
\end{abstract}

\section{INTRODUCTION}

Throughout Europe 15.000 young people die each year due to road accidents (Gadget Final Report, 2000). In many European countries traffic accidents are even the leading cause of death of young people. Regarding their fatality rate young drivers are over-represented in comparison to other age groups of drivers. The graphical representation of the fatality rate plotted against driver age shows a distinct U-shaped curve; fatality rates are highest for young drivers as well as 
for old drivers (70+ years old). The size of the problem makes clear that action is needed. It is important yet difficult to identify the causes of the high accident risk of young novice drivers, since many factors can be imagined to contribute to the high accident risk. Moreover almost none of these factors seem to operate alone.Novice drivers are strongly represented in accidents involving other vehicles. This holds especially for distance keeping and negotiating intersections. There is not a higher novice driver accident involvement during adverse weather conditions compared with experienced drivers. On the basis of accident analyses, motorway and urban driving appeared not problematic for novice drivers, but driving on secondary roads could be. 'Loss of control' is most frequently indicated as factor preceding the accident, which is an indication that either skills are not sufficiently crystallised, or of serious lapses in assessment of one’s own skills. .

Traditionally, driver training has focused on vehicle control skills and traffic rule knowledge without efforts to improve higher order skills. These higher order skills are included in driver training in many countries but rather in a theoretical way, included in textbooks, and is not covered in practical training. The reason is that this problem cannot be systematically handled in practical training, as on roads risky situations are scarce and usually avoided. However the causes of novice driver accidents can to a large extent be attributed to these higher order skills. Therefore it is clear that there is a pressing need for developing a new driver training methodology, which takes in account the typical novice driver accidents, and includes the use of new telematic aids in order to enable the simulation of situations addressing higher order skills.

\section{NOVICE DRIVERS TRAINING NEEDS}

One of the objectives of TRAINER were to identify from the existing literature the problems of novice drivers in performing particular driving tasks, finally the hierarchical model of driving behaviour of the European project GADGET was used. The following four levels are described by Keskinen (1996) and were later also applied in the EU-project GADGET (Hatakka et al. 1999): (1) Goals for life and skills for living, (2) Goals and context of driving (3) Mastering traffic situations (4) Vehicle manoeuvring. A safe driver is, however, not only skilled but usually also aware of risks and of own abilities and preconditions. This awareness even allows him or her to compensate for a lack of skills. In order to cover these different dimensions the matrix includes, independent of the three earlier levels, the dimensions: (1) Knowledge and skills, (2) Risk increasing factors, (3) Self assessment.

Obviously, the training of basic vehicle handling skills is basic to driver training. Driving simulators are important, novel devices for training the very first steps of vehicle handling. The advantages are not only safety related - trainees can learn these skills without endangering themselves or other road users - but are also associated with ecological and economical issues: Simulators are absolutely exhaust-free and as fuel is not consumed simulators may be relatively cheap in the end. Simulators also offer the possibility for trainees to learn, without any risk due to distraction, to handle new technical devices like Automatic Cruise Control (ACC) and AntiBlocking System (ABS). Trainees should be aware that they might adapt their behaviour because they feel safe with such car control support systems. It also appeared that trainees should experience different types of cars in the simulator (with respect to, e.g., size, front vs. rear wheel), because trainees may have difficulties transferring their skills to the other cars that they drive later. As performance feedback can be easily presented in simulators, trainees can train risk-related skills, such as speed estimation of speed and Time-to-Collision (TTC). In the 
simulator, they can evaluate their skills by comparing their own decisions and estimates with normative decisions and estimates and perceive the eventual consequences of their behaviour. Drivers have to know how speed influences certain parameters of visual perception, which are important prerequisites for a safe performance of all manoeuvring tasks. The trainees should understand the relationship between reaction type, braking and total stopping distance.

Various sources in the literature emphasise that learning to interact with other road users (i.e., manoeuvring skill) does not contribute to safe driving and reduction of accident rates of novice drivers. Both the literature and the experts suggest that scanning and anticipating skills play a more important role in safe driving. Therefore training of particular manoeuvring skills should also include training of perceptual skills relevant to the particular tasks. Furthermore, drivers appear to underestimate the time needed for many manoeuvring tasks, like overtaking, merging, lane changing, etc. This could be done with simulators alike as performance can be monitored and evaluated in a more objective manner than in real cars. Another main advantage of simulators, compared with real cars, is that trainees can experience scenarios that are too dangerous to create on the road, and that they can train cognitive skills without having automated their manoeuvring skills yet. Attempts to teach trainees safe-driving strategies during training often failed, probably because the information processing capacity of novice drivers is already overloaded by vehicle control and interacting with other traffic participants: Trainees have to make conscious decisions for every move and every action they take, so they are not able to use improvements of defensive or risk minimising strategies. Automatic Traffic Generation and Autonomous Driver models reproduce the circumstances in real traffic, and enable the users to repeat and therefore train certain tasks in changing environments with varying risk and different road users with variable behaviour. The influence of distractors (e.g., in-car: mobile phone, driver support systems, out of the car: ambulance horns) on attention and behaviour to cope with them could also be highlighted using simulators without real risk. Through the combination of opportunity to practice and obtaining feedback on those skills trainees can come to their own understandings of how cues in traffic and outcome are related. For all manoeuvring tasks it is important to state that simulators should be used only for a limited time. Trainees should not automatise skills in an artificial environment. For that reason, simulator training should be followed by real car training sessions. The training curricula should combine specific simulator scenarios with actual car driving. However, risky driving behaviour results not only from poor perception, but also from overestimation of one's own skills. In order to increase driving skills without increasing the confidence in these skills the manoeuvring component should not be overemphasised.

Many authors of road safety literature emphasise, that it is not crucial for safety how skilled a driver is, but to what extent drivers use their skill in driving safely. There are certain attributes of young people, which influence and/or cause their involvement in accidents. Trainees should learn that certain factors as lifestyle, social background, gender, age and other individual preconditions have an influence on attitudes, driving behaviour and accident involvement. Young drivers often have risky habits (e.g. testing limits of own skill), safety-negative motives (like competing or pleasure), and are prone to social pressure by peers (use of alcohol and drugs etc.). Exercises should be developed to make trainees aware that assessment of their own abilities to negotiate critical situations may be false, especially in the beginning. Driver education has to make them realise their own personal tendencies (risky habits, safety-negative motives). Proposals for appropriate educational methods derived from the literature are feedback during training, self-assessment tools like questionnaires and scales, discussion with other youngsters 
about personal experiences and attitudes, and evaluations made by instructors or examiners. Finally, it should be kept in mind that every training of manoeuvring skills (and probably this is also relevant for cognitive skills like e.g. hazard perception) may result in overconfidence of young drivers. Therefore training safe driving strategies can only be successful if driver training covers the whole range of proposed contents and therefore should also include motivational and self-evaluative aspects.

\section{SCENARIOS DEVELOPMENT ESTRATEGY}

A total of approximately 100 scenarios for application in the different simulation environments are developed, which addresses the most important needs of learner drivers. The scenarios have been structured in accordance with the four hierarchical levels of the GADGET-matrix.

\section{Example of scenario for level 2: Gap acceptance, simulator application}

\section{Description of the problem that needs to be addressed}

Novice drivers lack experience in judging the speed of other cars. This has been shown to be the case in car following (see also below), but is likely to play a role in gap acceptance tasks as well. A fair amount (14-24\%) of accidents happen on junctions and is related to failure to yield. The accepted gap is inversely related to age, which supports the idea that young novice drivers accept higher risk in this situation.

\section{Definition of the aim of the scenario}

The trainee shall practice estimating gaps and calibrate gap acceptance in meeting traffic when turning left, both to avoid driving when the gap is too small and to avoid waiting unnecessarily long before turning

\section{Description of the situation that should be simulated}

The scenario is set in a built up area, speed limit typically $50 \mathrm{~km} / \mathrm{h}$. There is one car in front, at some 100 metres (approx. 4 seconds time headway when driving $50 \mathrm{~km} / \mathrm{h}$ ). The car approaches a junction and has to turn left. At that time cars from the opposite direction arrive at the junction. All these cars are heading straight on. The trainee's car is indicated with a T.

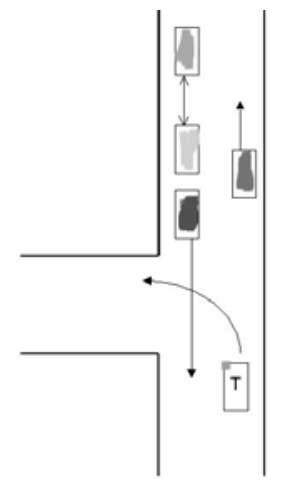

The gap between the cars coming from the opposite direction increases by 1 second time headway. So, the distance between the first and second car is 1 second, between the second and third is 2 seconds, and so on (up to a maximum of 15 seconds). If the gap is less than 5 seconds, when turning left, the driver is taking unnecessarily high risk.

Possible ways to show this are:

1. Flashing headlights of the approaching car

2. Helicopter view of the situation, demonstrating that the approaching car has to slow down.

As the size of the approaching vehicle, as well as whether it drives with headlights on, affects the accepted gap and these variables can be used to create variations to the above scenario. 


\section{Success criterion}

The trainee accepts the 5 seconds rule for gap acceptance and manages to use this criterion in own choice.

\section{A reference to which cell in the GADGET-matrix the scenario applies}

Mastering traffic situations, knowledge and skill, risk increasing factors and self assessment

\section{TRAINER SIMULATION TOOLS REQUIREMENTS}

By means of modular design the Consortium have developed a low cost stationary driving simulator for driving schools, able to support the driver in understanding basic control actions and to provide some didactic feedback. Based on it, a mean cost semi-dynamic driving simulator has also been built; to be used in selected driving schools and/or assessment centres in order to assist drivers with specific difficulties or particular driver cohorts. These include high risk novice drivers, people with higher cognitive problems (i.e. elderly, etc.). Both simulators has been developed on a modular basis, based on existing components from state of the art driving simulators, developed and currently marketed.

The simulators developed are used as:

- Driver's training tool for complex (and safety critical) traffic scenarios in driving schools.

- Driver's assessment tool (for all drivers or particular driver subgroups - i.e. elderly and cognitive disabled, standard drivers with knowledge acquisition problems or after serious accidents for re-training), installed at central assessment points.

And main objectives for the development of the simulators were:

- To develop appropriate scenarios to support driver training and assessment by the use of simulators.

- To develop a low cost driving simulator to support driver training in tactical and control tasks, according to the Michon model.

- To develop a mean cost driving simulator with high reliability for support and assessment of particular drivers cohorts.

4 prototypes have been developed, of each type, so as to be used in each of the 4 Pilot sites (Belgium, Greece, Spain and Sweden).

The most interesting thing in the development of the simulator, and in fact, in the software development, was the meticulous selection of the scenarios, which let to the driving school instructor to have a very powerful tool to develop a specific training program, which ranges a lot of different real situations related to the driving safety. 


\section{ACKNOWLEDGMENTS}

The project "System for Driver Training and Assessment using Interactive Evaluation Tools and Reliable Methodologies” (TRAINER) is funded by EU GROWTH N GRD 1-1999-10024. Key Action "Sustainable Mobility and Intermodality” of “Competitive and Sustainable Growth" V ${ }^{\text {th }}$ EU programme.

\section{REFERENCES}

1. Gadget Final Report (2000). Investigations on Influences upon Driver Behaviour; Safety Approaches in Comparison and Combination.

2. Hall, J. \& West, R. (1996). The role of formal instruction and informal practice in learning to drive. Ergonomics, 39, 693-706.

3. Hatakka, M., Keskinen, E., Gregersen, N. P., Glad, A, Hernetkoski, K. (1999). Results of EU-project GADGET, Work Package 3. In. S. Siegrist (ed): Driver Training, Testing and Licensing - towards theory based management of young drivers' injury risk in road traffic. BFU-report 40. Schweizerische Beratungsstelle für Unfallverhütung.

4. Keskinen, E. (1996). Why do young drivers have more accidents? Junge Fahrer und Fahrerinnen. Referate der Ersten Interdisziplinären Fachkonferenz 12-14. December 1994, Köln. Berichte der Bundesanstalt für Strassenwesen, Mensch und Sicherheit, Heft M 52.

5. Michon, John A. (1985). A critical view of driver behavior models: what do we know, what should we do?, in Evans, L. \& Schwing, R.C. (eds), Human Behavior and Traffic Safety, 485-520. 